\title{
Topographical Organization of the Entorhinal Projection to the Dentate Gyrus of the Monkey
}

\author{
M. P. Witter, ${ }^{1}$ G. W. Van Hoesen, ${ }^{2}$ and D. G. Amaral ${ }^{3}$ \\ 'Department of Anatomy and Embryology, Vrije Universiteit, 1081 BT Amsterdam, The Netherlands, ${ }^{2}$ Departments of \\ Anatomy and Neurology, University of lowa, College of Medicine, lowa City, lowa 52242, and ${ }^{3}$ The Salk Institute for \\ Biological Studies, San Diego, California 92128
}

The topographic organization of the projections from the entorhinal cortex to the dentate gyrus in the macaque monkey was studied with anterograde and retrograde tracing methods. Injections of WGA-HRP or the fluorescent retrograde tracers, Fast blue and Diamidino yellow, were placed at various levels along the rostrocaudal axis of the dentate gyrus and hippocampus. In 5 experiments the fluorescent dyes were injected at 2 rostrocaudal levels of the same dentate gyrus. Labeled neurons were observed mainly in layers II and III of the entorhinal cortex, though some were also seen in layers V and VI. The labeled layer II cells resulting from each of the tracer injections were located throughout much of the rostrocaudal extent of the entorhinal cortex, though they tended to have a more limited distribution in the transverse or mediolateral axis. Injections of retrograde tracers located caudally in the dentate gyrus resulted in a rostrocaudally oriented zone of labeled cells that was situated laterally in the entorhinal cortex adjacent to the rhinal sulcus. The zone of labeled cells was not oriented strictly parallel to the rhinal sulcus since at caudal levels it extended medially to encompass the full transverse extent of the most caudal portion of the entorhinal cortex. When injections were placed more rostrally in the dentate gyrus and hippocampus, the rostrocaudally oriented zone of labeled cells was situated more medially in the entorhinal cortex. Anterograde tracing experiments using ${ }^{3} \mathrm{H}$-amino acid injections into different rostrocaudal and mediolateral positions of the entorhinal cortex confirmed the organization demonstrated by the retrograde tracers and further indicated that the entorhinal fibers terminate in the outer two-thirds of the molecular layer of the dentate gyrus. Unlike in the rat, where the entorhinal termination zone in the molecular layer is clearly bilaminate, projections from all portions of the entorhinal cortex appeared to terminate more diffusely throughout the outer two-thirds of the molecular layer.

\footnotetext{
Received Feb. 19, 1988; revised May 11, 1988; accepted May 13, 1988

We would like to thank Ms. Janet Weber for her help in preparing much of the histological material, Mr. Dirk de Jong, Mr. P. Reimann, and Mr. Kris Tulock for help with the illustrations, and Mrs. N. Nauta-Kaat for help in typing the manuscript. This work was supported by NIH Grants NS 16980 to D.G.A. and 14944 to G.W.V.H. M.P.W. was supported by grants from the Van Coeverden Adriani Foundation and the Netherlands Organization for the Advancement of Pure Research (ZWO).

Correspondence should be addressed to Menno P. Witter, Department of Anatomy and Embryology, Vrije Universiteit, van der Boechorststraat 7, $1081 \mathrm{BT}$ Amsterdam, The Netherlands.

Copyright (c) 1989 Society for Neuroscience $0270-6474 / 89 / 010216-13 \$ 02.00 / 0$
}

The results of the present study indicate that rostrocaudally oriented zones of cells that cut across several cytoarchitectonic subdivisions of the entorhinal cortex give rise to topographically organized projections to the dentate gyrus. Cells located laterally in the entorhinal cortex project to caudal levels of the dentate gyrus, whereas progressively more medially situated cells project to progressively more rostral parts of the dentate gyrus.

Behavioral data in primates, including man, indicate that the hippocampal formation plays an important role in memory processes (Scoville and Milner, 1957; Mishkin, 1978; Zola-Morgan and Squire, 1986; Zola-Morgan et al., 1986). While the precise mechanism by which it contributes to the long-term storage of information is not known, it is clear that the hippocampal formation is not the sole repository of memory. Information from human amnesic cases (Scoville and Milner, 1957; Zola-Morgan et al., 1986), in fact, indicates that hippocampal dysfunction contributes primarily to an anterograde memory impairment with substantially preserved premorbid or retrograde memory. The implication of these data is that the hippocampal formation has access to cortically processed sensory information, performs some operations with the information, and then interacts with storage sites in other brain regions, including the cortex.

Anatomical studies in a variety of mammals have demonstrated that most of the cortical sensory information that reaches the hippocampal formation enters via the entorhinal cortex. In the rat, cat, and monkey, the entorhinal cortex receives inputs primarily from polysensory cortical areas either directly or by way of the adjacent perirhinal cortex (Jones and Powell, 1970; Van Hoesen and Pandya, 1975a; Van Hoesen et al., 1975; Deacon et al., 1983; Room and Groenewegen, 1986; Insausti et al., 1987). This highly processed sensory information is subsequently transmitted to the dentate gyrus and hippocampus by way of the "perforant pathway" (Van Hoesen and Pandya, 1975b; Steward, 1976; Van Hoesen, 1982; Witter et al., 1986; Insausti et al., 1987).

The perforant pathway projection to the dentate gyrus in the cat and the rat has been studied in detail (Steward, 1976; Wyss, 1981; Witter and Groenewegen, 1984). This projection arises primarily from layer II cells of the entorhinal cortex while projections to the hippocampus and subiculum originate in layer III and deeper layers (Steward and Scoville, 1976; Witter and Groenewegen, 1984). Earlier studies of the perforant pathway projection in the monkey (Van Hoesen and Pandya, 1975b; Van 
Hoesen, 1982) indicated that its general organization resembles that in the rodent. However, more recent studies in the rat and the cat have provided new insights into the topographic organization of the perforant pathway (Ruth et al., 1982; Witter and Groenewegen, 1984; Van Groen and Lopes da Silva, 1985). These studies indicate that cells in different mediolateral zones of the entorhinal cortex project to different septotemporal levels of the dentate gyrus. Layer II cells located near the rhinal sulcus (in both the medial and lateral subdivisions of the entorhinal cortex) project predominantly to the septal part of the dentate gyrus, whereas cells located progressively more medially in the entorhinal cortex project to more temporal parts of the dentate gyrus.

The entorhinal cortex of the macaque monkey has recently been parcelled into 7 cytoarchitectonically distinguishable subdivisions (Amaral et al., 1987). These are arranged from rostral to caudal in the entorhinal cortex. In the present studics we sought to determine whether the monkey perforant pathway projection to the dentate gyrus is organized in relation to the cytoarchitectonic subdivisions of the entorhinal cortex (i.e., do different subdivisions give rise to different perforant pathway components?) or in a manner similar to the rostrocaudally oriented zones described previously in the cat (Witter and Groenewegen, 1984) (i.e., does the origin of the perforant pathway projection to different portions of the dentate gyrus encompass portions of several cytoarchitectonic divisions?). Since there is no detailed analysis of the topography of the monkey perforant pathway currently available, we have conducted a series of retrograde and anterograde experiments to define its organization.

\section{Materials and Methods}

In the present study, 26 Macaca fascicularis monkeys and 13 rhesus (Macaca mulatta) monkeys of either sex were used. The animals weighed approximately $3 \mathrm{~kg}$ at the time of surgery.

Retrograde tracer studies. Retrogradely transported tracers were injected into the dentate gyrus and hippocampus of 10 fascicularis monkeys and 1 rhesus monkey. In 5 experiments with the fascicularis monkeys, injections were placed bilaterally, and 2 fluorescent tracers [Fast blue (FB) and Diamidino yellow (DY)] were injected at different rostrocaudal levels on each side. In the remaining experiments, animals received single injections of either HRP, WGA-HRP, or one of the fluorescent tracers into the dentate gyrus.

Anterograde tracer studies. In a second series of experiments, single injections of ${ }^{3} \mathrm{H}$-amino acids were placed into the entorhinal or perirhinal cortices of 16 fascicularis and 6 rhesus monkeys. One of the fascicularis monkeys received bilateral injections of the entorhinal cortex. The autoradiographic cases were supplemented with material from 6 rhesus monkeys who had received small, subpial ablations, and their brains were subsequently processed to demonstrate the distribution of anterograde degeneration using silver impregnation methods. This material was used in an earlier description of entorhinal efferents (Van Hoesen and Pandya, 1975b), and the procedures are described in detail in that paper.

Surgical and histological procedures. Animals were preanesthetized with ketamine hydrochloride $(8 \mathrm{mg} / \mathrm{kg}$, i.m.) followed by Nembutal $(25$ $\mathrm{mg} / \mathrm{kg}$, i.p.) and mounted in a Kopf stereotaxic apparatus. Using sterile procedures, the skull was exposed, and a small burr hole was made at a site appropriate for the injection as determined from the atlas of Szabo and Cowan (1984). In some cases, a tungsten microelectrode was lowered through the intended injection site, and single extracellular unit responses were recorded along its trajectory. This procedure allowed us to determine more precisely the appropriate dorsoventral coordinate for injection of tracer into the dentate gyrus or entorhinal cortex. Following injection of the tracer, the pipette or syringe was slowly withdrawn, and the wound was cleaned and sutured. A prophylactic regimen of antibiotics was administered during the survival period.

In most cases, the tracer substances were dispensed through glass micropipettes using air-pressure pulses (Amaral and Price, 1983), but some of the dye injections were placed with a $1 \mu 1$ Hamilton syringe. For the dye injections, solutions of $2 \%$ DY or $3 \%$ FB were used. At each injection site, $200 \mathrm{nl}$ of the dye was dispensed over $20 \mathrm{~min}(2$ injections of $100 \mathrm{nl}$ were placed with $1 \mathrm{~mm}$ vertical separation). For the experiments in which 2 injections were made into different levels of the dentate gyrus $(n=5)$, the FB was placed at the more rostral of the 2 sites, and the procedure was then repeated with the DY at the more caudal site. Animals were allowed to survive for 7-14 $\mathrm{d}$ and were then deeply anesthetized and perfused transcardially with $4 \%$ paraformaldehyde in $0.1 \mathrm{M}$ phosphate buffer. After 3-5 d of postfixation in the same fixative and cryoprotection with $20 \%$ glycerol in $0.1 \mathrm{M}$ phosphate buffer, the brains were sectioned at $50 \mu \mathrm{m}$ on the freezing, sliding microtome. Two adjacent 1-in-5 series of sections were immediately mounted on slides and stored dessicated at 4 and $20^{\circ} \mathrm{C}$ until analyzed with a Leitz Dialux-20 microscope equipped for fluorescence microscopy. An adjacent 1 -in-5 series of sections was stained with thionin to allow the determination of cytoarchitectonic boundaries of the entorhinal cortex. In addition to the experiments using the fluorescent retrograde tracers, injections of either WGA-HRP or HRP were made into the dentate gyrus and hippocampus of 6 animals, either unilaterally $(n=5)$ or bilaterally $(n=1)$. These injections consisted of 50-100 $\mathrm{nl}$ of either a $1 \%$ solution of WGA-HRP or $30 \%$ HRP. Animals were allowed to survive for $2 \mathrm{~d}$, and the brains were processed according to the procedure of Mesulam (1976) as previously described (Amaral and Cowan, 1980; Insausti et al., 1987).

The ${ }^{3} \mathrm{H}$-amino acid injections into the entorhinal cortex consisted of a single injection of $50-100 \mathrm{nl}$ of a 1:1 mixture of ${ }^{3} \mathrm{H}$-leucine and ${ }^{3} \mathrm{H}$ proline (concentrated to $100 \mu \mathrm{Ci} / \mu \mathrm{l}$ ). Animals were allowed to survive for 7-14 $\mathbf{d}$ and then deeply anesthetized and perfused as above. Sections were cut at $30 \mu \mathrm{m}$ and processed according to the protocol of Cowan et al. (1972) for the autoradiographic demonstration of the anterogradely transported isotope.

Analysis of material and preparation of summary diagrams of the entorhinal cortex and dentate gyrus. The distribution of retrogradely labeled cells in the entorhinal cortex was analyzed for each experiment using a computer-aided $X-Y$ plotting system. The position of each retrogradely labeled cell was plotted for every section in the 1 -in- 5 series through the entorhinal cortex. The plots of retrogradely labeled cells were then used to produce 2-dimensional surface maps of the entorhinal cortex in which the position of each section and the labeled neurons that it contains are represented. This procedure allowed the full rostrocaudal and mediolateral extent of the retrogradely labeled layer II cells to be easily appreciated. The method for construction of the 2-dimensional entorhinal maps (Fig. 1) was described in a previous report (Amaral et al., 1987). A standard 2-dimensional map of the fascicularis entorhinal cortex was also used to illustrate the positions and extents of the ${ }^{3} \mathrm{H}$-amino acid injections (Fig. 5).

For the autoradiographic experiments, the distribution of anterogradely transported ${ }^{3} \mathrm{H}$-amino acids in the dentate gyrus was analyzed using dark-field illumination. The position and density of labeled fibers and terminals was plotted on representative camera lucida drawings of sections through the full rostrocaudal extent of the dentate gyrus. To facilitate the comparison of the distributions of terminal labeling between the various experiments, the position and relative density of silver grains in each experiment were redrawn onto a standardized schematic representation of the dentate gyrus. This (Fig. 1) was made by drawing the outline of a dissected hippocampus of the fascicularis monkey; the resultant template provided a convenient way of representing terminal distribution over the full rostrocaudal extent of the dentate gyrus. By positioning several of these templates in close proximity to cach other (as in Fig. 5), differences in the overall longitudinal distribution of projections can be easily appreciated relative to the locations of injection sites.

\section{Results}

\section{Nomenclature}

Terminology for the various subdivisions of the entorhinal cortex and the criteria used for establishing the boundaries between the subdivisions have recently been described (Amaral et al., 1987). While this description was initially applied only to the fascicularis brain, analysis of frontal sections through the rhesus monkey entorhinal cortex stained with Nissl or fiber methods, by the Timm's sulfide silver method for the presence of heavy 


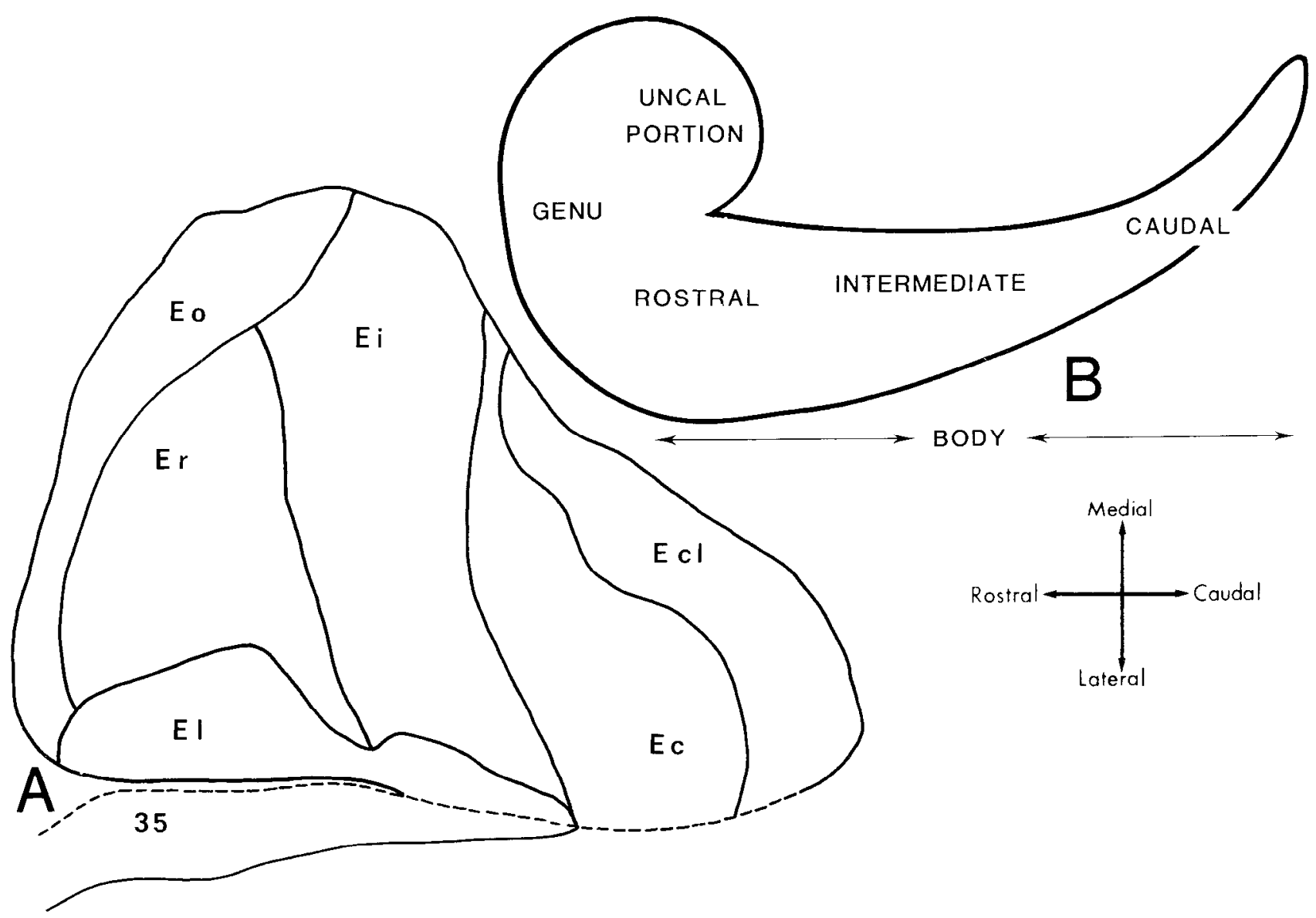

Figure 1. A, An "unfolded" 2-dimensional map of the surface area of the entorhinal cortex and adjacent area 35 . The rhinal sulcus is represented as a dashed line, and all fields are enclosed by solid lines (after Amaral et al., 1987). B. Schematic representation of the dentate gyrus. For descriptive purposes 3 regions of the dentate gyrus (uncal portion, genu, and body) are indicated.

metals, or for the presence of acetylcholinesterase indicates that the nomenclature is equally applicable to this species. The entorhinal cortex of both species is subdivided into 6 parts (Fig. 14 ) that are arranged from rostral to caudal. Most rostrally is the olfactory division (Eo), so named because it is the only entorhinal field that receives a direct projection from the olfactory bulb. Eo is bordered caudally by the lateral (El) field, that forms the medial bank of the rhinal sulcus, and the rostral field $(\mathrm{Er})$. The intermediate field (Ei) is caudal to Er and is separated from the rhinal sulcus by a caudal extension of El. The subdivision of $\mathrm{El}$ into a rostral and caudal part $\left(\mathrm{El}_{\mathrm{r}}\right.$ and $\mathrm{El}_{\mathrm{c}}$ in Amaral et al., 1987) has been omitted in the present study. The 2 remaining fields extend across the full transverse distance of the caudal entorhinal cortex. The caudal subdivision $(\mathrm{Ec})$ borders Ei and El rostrally, and is caudally replaced by the caudal limiting subdivision $(\mathrm{Ecl})$. For a more detailed description of the organization of these fields see Amaral et al. (1987).

As it became clear that the entorhinal projection to the dentate gyrus is topographically organized in relation to the rostrocaudal or longitudinal extent of the dentate gyrus, it became necessary to adopt a nomenclature that would allow reference to be made to different positions along this axis. The terminology of Demeter et al. (1985) was appropriate for this purpose. In this nomenclature, the dentate gyrus and hippocampus can be characterized as having 3 major regions: the uncal portion, the genu, and the body (Fig. 1B). The uncal portion is the most medial part of the hippocampus that is located at the rostral limit of the temporal horn of the lateral ventricle where the hippocampus and dentate gyrus bend medially and then caudally and form part of the uncus. The uncal portion of the dentate gyrus and hippocampus constitutes the most rostral extent of these fields. The genu is the flexed region where the uncal portion is continuous with the main portion or body of the hippocampus. In frontal sections it is characterized by a ring-like arrangement of the granule cell layer of the dentate gyrus. The body of the hippocampus and dentate gyrus makes up the major portion of these fields and can be subdivided into rostral, intermediate, and caudal parts. The rostral part of the body of the hippocampus is essentially the portion associated with the optic tract, whereas the intermediate part is associated with the lateral geniculate nucleus and the caudal part extends caudal to the lateral geniculate nucleus. We should emphasize that these are purely arbitrary terms that we are using to facilitate the description of projections to different rostrocaudal levels of the hippocampus and dentate gyrus. There are no obvious cytoarchitectonic differences at different rostrocaudal levels of the hippocampus or dentate gyrus that would allow assignment to one of the portions named above.

\section{Retrograde tracer experiments}

The cells of origin for the perforant pathway projection to different levels of the dentate gyrus were determined by making 
discrete injections of retrograde tracers at different levels along the rostrocaudal axis of the dentate gyrus. In conducting these studies we adopted 2 strategies that not only facilitated the analysis of the topography of the perforant pathway projection but limited the number of animals necessary for the study. The degree to which projections to different rostrocaudal levels of the dentate gyrus arise from different portions of the entorhinal cortex was most easily assessed in experiments in which $2 \mathrm{flu}$ orescent dyes were placed into different rostrocaudal levels of the same dentate gyrus. By plotting the distribution of singleand double-labeled cells in the entorhinal cortex, substantially greater topographic information could be attained than in the single-injection experiments. Given the potential fiber of passage problem that might arise with the use of retrograde tracers, our decision to use this strategy was prompted by considerations of the trajectory of perforant pathway fibers to the dentate gyrus. From the analysis of the anterograde tracing experiments we conclude that perforant pathway fibers project to the ipsilateral dentate gyrus by way of the so-called angular bundle that lies ventromedial to the dentate gyrus. Perforant pathway fibers travel in this bundle and only enter hippocampal subfields at, or very close to, their level of termination. Thus, small retrograde tracer injections that do not impinge on the angular bundle are not likely to significantly involve perforant pathway fibers that are destined for other levels of the dentate gyrus and only those entorhinal cells that project to the area injected will be retrogradely labeled. A second procedure used to increase the number of observations was the bilateral placement of retrograde tracers in the dentate gyrus. The known organization of the hippocampal commissural system makes this an acceptable procedure. Thus, the commissural projection from the entorhinal cortex to the dentate gyrus is very light; it arises only from the caudal pole of the entorhinal cortex, and it terminates only in the most caudal part of the contralateral dentate gyrus (Amaral et al., 1984). None of our retrograde tracer injections involved the caudal portion of the dentate gyrus that receives commissural projections.

All retrograde tracer injections of the dentate gyrus resulted in the labeling of neurons in the entorhinal cortex. Conversely, all parts of the entorhinal cortex project to the dentate gyrus. When the retrograde tracer was confined to the dentate gyrus and did not involve the hippocampus or subiculum, labeled cells were observed primarily in layer II of the entorhinal cortex though there were generally at least some labeled cells located in layer $\mathrm{V}$ as well. In most of the experiments, the injection of tracer had at least minor involvement of the hippocampus or subiculum and in these cases labeled cells were observed mainly in layers II and III and to a lesser extent in layers V and VI. While additional studies of the laminar origin of the monkey perforant pathway projection must be conducted, it appears that, as in the rat and cat, the projection to the dentate gyrus arises mainly from layer II. Thus, the following topographic summaries will be based only on the distribution of labeled layer II cells. We will begin our account with a description of the double fluorescent dye experiments in which the topographic organization of the perforant pathway projection is clearly illustrated. We will not describe redundant cases but should emphasize that the patterns described below have been observed in several retrograde experiments.

In experiment M-6-86 (Fig. 2), 2 dye injections were placed into the left dentate gyrus. The FB injection involved the lateral portion of the genu and the DY injection involved caudal levels of the intermediate and rostral levels of the caudal portions of the body of the dentate gyrus (Fig. $2 A$ ). As represented in the unfolded map of the entorhinal cortex in Figure $2 A$, the DY injection resulted in a rostrocaudally oriented zone of labeled layer II neurons (represented as red dots), that bordered the rhinal sulcus and extended throughout the rostrocaudal extent of $\mathrm{El}$ and the adjacent parts of $\mathrm{Er}$ and $\mathrm{Ei}$. The zone of labeled cclls widencd in the caudal portion of $\mathrm{Ei}$ and continued to expand in the caudal and caudal limiting fields so that at the caudal pole of the entorhinal cortex, labeled cells occupied its full transverse extent. Cells labeled from the more anteriorly placed injection of FB (represented as black dots) formed a more medially located zone. This zone of labeled cells involved the midtransverse position of $\mathrm{Er}$ and $\mathrm{Ei}$, the medial half of $\mathrm{Ec}$, and the rostromedial part of Ecl. There was a fairly narrow region of overlap of the zones of labeling from each injection. This border region contained single-labeled cells of both types as well as some double-labeled neurons (Fig. 2B). A few labeled cells were observed laterally in Eo, and no labeled cells were present in the medial portions of Eo, Er, and Ei.

As in experiment M-6-86, case M-2-87 (Fig. 3) received injections of FB and DY into the left dentate gyrus. The DY injection involved the lateral part of the genu in a position similar to that of the FB injection in M-6-86. By comparing the black dots in Figure $2 A$ with the red dots in Figure 3, it can be seen that the distribution of retrogradely labeled cells that projected to this region was similar in both experiments. The injection of FB in the left side of experiment M-2-87 involved the medial part of the genu and the rostral portion of the uncal part of the dentate gyrus and hippocampus. Note that this injection led to a greater number of retrogradely labeled cells in Eo and to a medial shift in the distribution of labeled cells in the other entorhinal fields. In contrast to the pattern in M-686 , there were very few labeled cells in $\mathrm{El}$ and in the lateral portions of Ec and Ecl. As in M-6-86, there was a zone of overlap of the DY and FB labeled cells in which some cells were double labeled.

In experiment $\mathrm{M}-5-87,2$ dyes were injected into the dentate gyrus on both sides of the brain. On the right (Fig. 4), the FB injection involved the most caudolateral extent of the uncal portion and the adjacent rostral body of the dentate gyrus. The DY injection was located in the caudal portion of the body of the hippocampus, where it heavily involved the subiculum and more lightly involved the dentate gyrus. What distinguishes this case from $\mathrm{M}-6-86$ and $\mathrm{M}-2-87$ is that the $\mathrm{FB}$ injection in the uncal portion of the dentate gyrus led to greater labeling in the olfactory portion of the entorhinal cortex. Moreover, the zone of retrogradely labeled cells in $\mathrm{Er}$ and $\mathrm{Ei}$ was more medially situated than in experiment M-2-87. Consistent with the caudal placement of the DY injection in M-5-87, the retrogradely labeled layer II cells were located in the extreme lateral portion of the entorhinal cortex.

In experiment $M-3-87$, the fluorescent dye injections attained positions very similar to those in $\mathrm{M}-6-86$. The main difference was that the DY injection was more rostrally positioned in the body of the hippocampus. The change in retrograde labeling pattern consisted of a medial shift in the distribution of DYlabeled cells in Ec and Ecl. The left side of experiment M-5-87 also had 2 injections into the hippocampus and dentate gyrus. In this case the FB injection was focused in the caudal part of the rostral portion of the body and the DY injection was in the caudal part of the body. The DY injection led to heavy labeling 


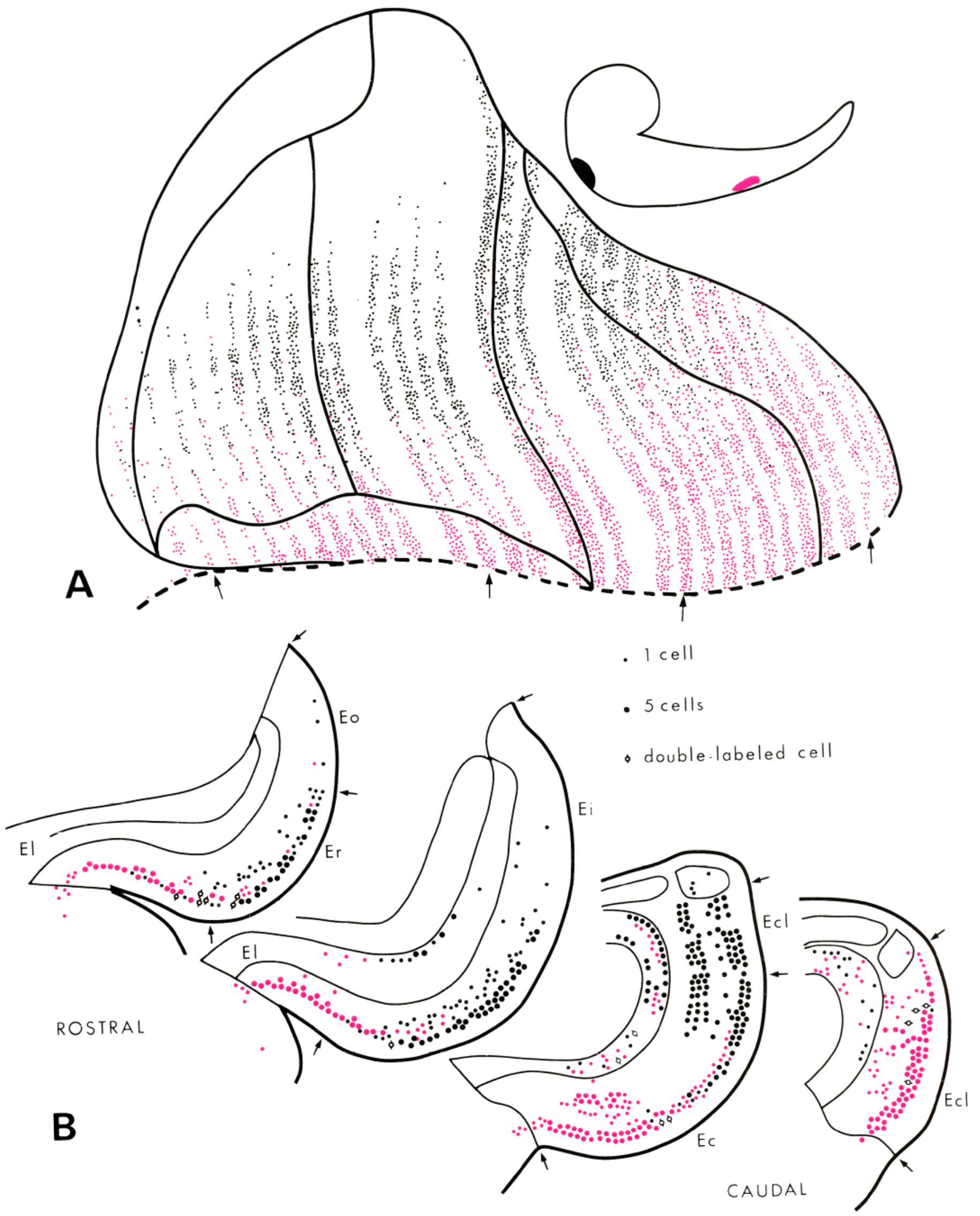

Figure 2. A, Distribution of retrogradely labeled layer II cells in experiment M-6-86 is plotted on an unfolded map of the entorhinal cortex. Note that the labeled cells in each section that was analyzed have been charted directly on the unfolded map. Each stripe thus represents an actual section 


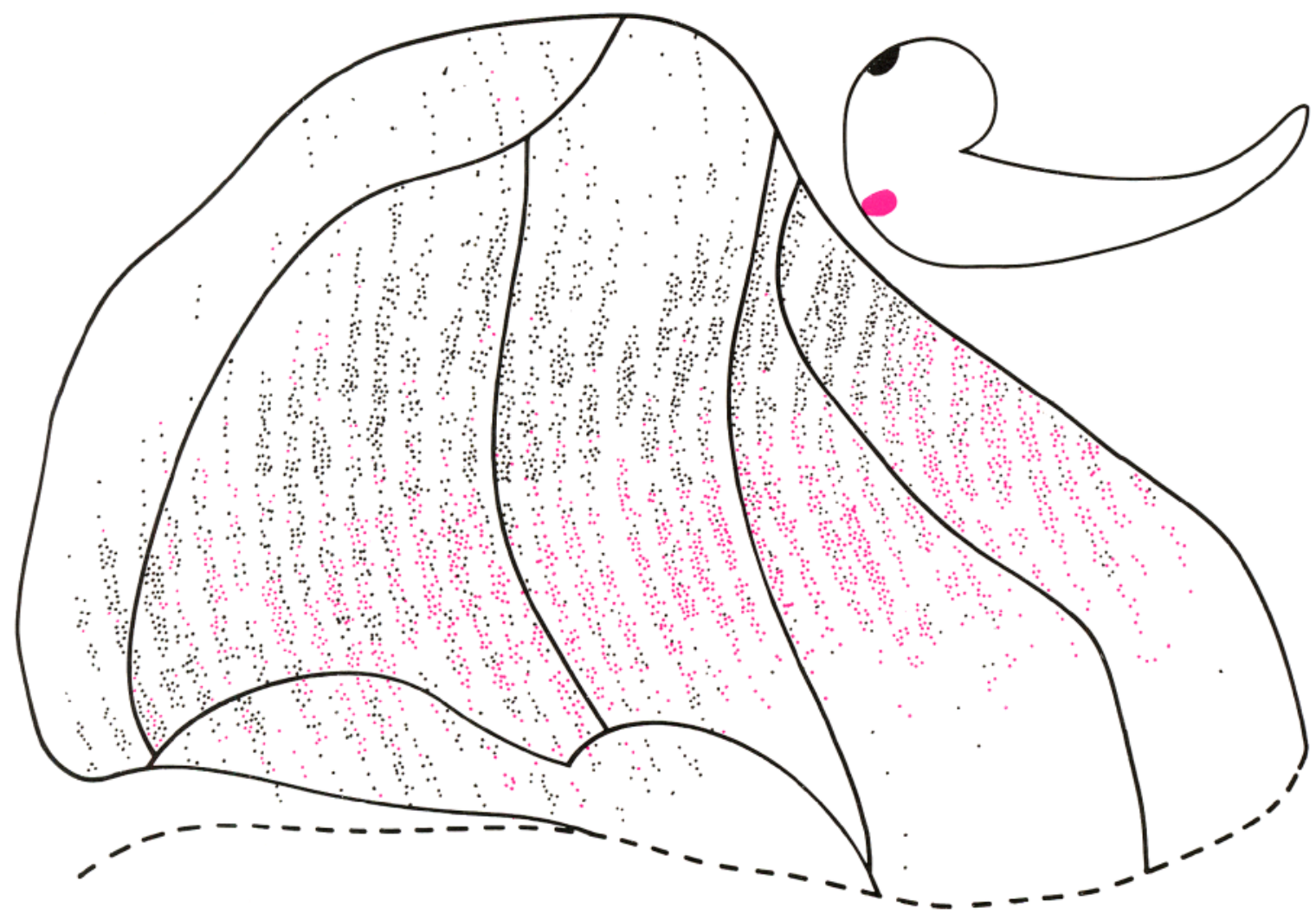

Figure 3. Distribution of retrogradely labeled layer II cells in case M-2-87, represented on an unfolded map of the entorhinal cortex. Labeled cells resulting from a DY injection laterally in the genu are plotted in red, while labeled cells following a FB injection medially in the genu are represented in black.

of the extreme lateral aspect of Ec and Ecl, whereas the FB injection led to a zone of labeling that was heaviest in a midtransverse position. There were several single dye and single WGA-HRP or HRP injections that involved the dentate gyrus and were available for analysis. These uniformly provided retrograde labeling patterns that were consistent with the cases described above.

These findings can be summarized as follows: injections of retrograde tracers that involve any rostrocaudal level of the dentate gyrus and hippocampus result in the labeling of zones of layer II cells that extend throughout much of the rostrocaudal extent of the entorhinal cortex. If the injection is placed caudally in the dentate gyrus, the zone of cells is located laterally in the entorhinal cortex along the rhinal sulcus. As the injection is placed at progressively more rostral levels, the zone of labeled cells tends to shift medially. When the injection is placed at the rostral limit of the dentate gyrus, in the uncal portion, labeled cells are numerous in the most medial aspects of Eo, Er, and Ei.

\section{Anterograde tracer experiments}

While the overall topographic organization of the entorhinal projection to the dentate gyrus is clearly demonstrated in the retrograde tracing experiments, these studies give no indication as to how the perforant pathway terminates in the dentate gyrus. To evaluate this aspect of the projection and to confirm the topographic organization indicated by the retrograde experiments, we analyzed the distribution of anterogradely transported ${ }^{3} \mathrm{H}$-amino acids resulting from injections into different portions of the entorhinal cortex.

Since the cytoarchitectonic organization of the entorhinal cortex in the fascicularis and rhesus monkeys is very similar and the pattern of perforant pathway projections to the dentate gyrus in the 2 species also appears to be comparable, we have combined the analysis of experiments for both species. Figure 5 summarizes the locations of the entorhinal injection sites for 14 experiments analyzed for this study. We have also presented schematic illustrations of the rostrocaudal distribution of anterograde labeling in the dentate gyrus for each of the experiments. The distribution and density of anterogradely transported label has been plotted as dots on the 2-dimensional representations of the dentate gyrus.

All of the ${ }^{3} \mathrm{H}$-amino acid injections into the entorhinal cortex resulted in prominent anterograde labeling in the molecular layer of the dentate gyrus. While there was additional heavy labeling of the subiculum and hippocampus, we will not describe

and the labeled cells that it contains (see also Figs. 3 and 4). Red dots represent DY-labeled neurons following an injection that involved the caudal part of the intermediate body of the dentate gyrus. Black dots represent FB-labeled cells resulting from an injection into the lateral part of the genu of the dentate gyrus. Double-labeled cells are not indicated (compare Fig. $B$ ). The template of the dentate gyrus shows the location of the 2 retrograde tracer injections. Arrows indicate the positions of the coronal sections illustrated in $B$. B. Line drawings of representative coronal sections through the entorhinal cortex of monkey M-6-86, illustrating the distribution of retrogradely labeled neurons. 


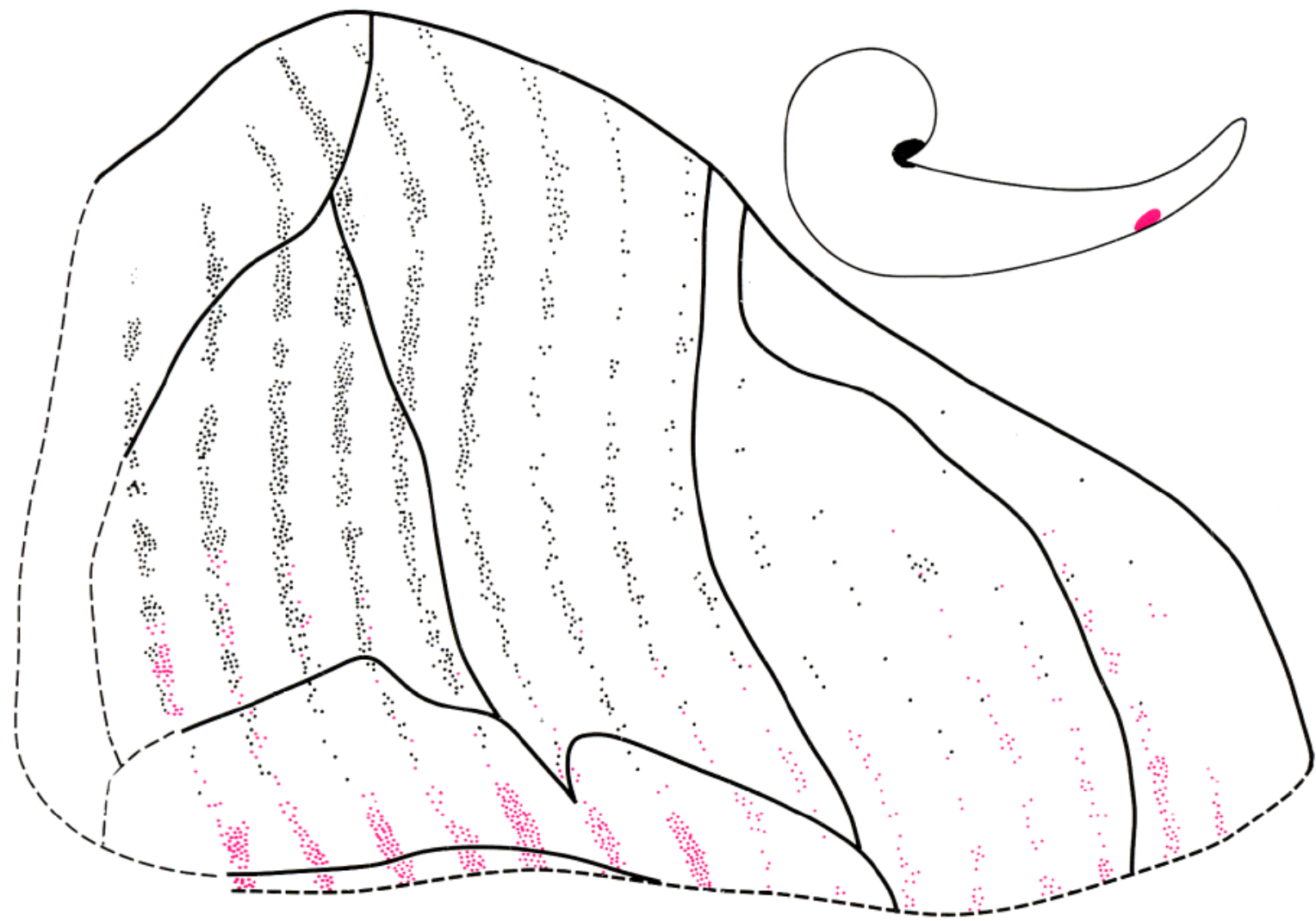

Figure 4. Distribution of retrogradely labeled layer II cells in case M-5-87, represented on an unfolded map of the entorhinal cortex. Labeled cells resulting from a DY injection in the caudal part of the body are plotted in red, while labeled cells following a FB injection in the caudolateral part of the uncal portion are represented in black. Dashed lines indicate rostral portion of the entorhinal cortex that was lost during processing.

these projections in this report. Unlike in the rat and cat, projections to the dentate gyrus were generally not confined to either the middle or outer third of the molecular layer. Only in case MPQ, which had the most rostral injection, was the terminal labeling restricted to the outer third of the molecular layer. In all other cases, the region of densest terminal labeling contained silver grains that were distributed over the entire outer two-thirds of the molecular layer. However, the laminar pattern of labeling was not homogeneous throughout the rostrocaudal extent of the dentate gyrus. Rostrally placed injections (i.e., those in $\mathrm{Er}$ and $\mathrm{Ei}$ ) had terminal distribution patterns that were generally biased towards the outer third of the molecular layer, whereas injections in Ec and Ecl had patterns of terminal distribution that preferentially labeled the middle third of the molecular layer.

The results of the retrograde tracing experiments indicated that rostrocaudally arranged zones of cells that are oriented obliquely to the axis of the rhinal sulcus project to different rostrocaudal levels of the dentate gyrus. The anterograde experiments clearly confirmed this organizational pattern. We will review the overall pattern of projections demonstrated by the autoradiographic experiments by referring to the schematic illustrations in Figure 5 in 2 ways. First, we will look at the terminal patterns of experiments with injections that are located at the same rostrocaudal level in the entorhinal cortex but in different mediolateral positions. The retrograde studies indicated that the more medial injections should generally lead to labeling of the rostral dentate gyrus, while the more laterally placed injections should preferentially label caudal levels. In a second comparison, we will compare 4 groups of injections that are located at different rostrocaudal levels in the entorhinal cortex but within the same mediolateral zone. The projection pattern for each experiment in these groups should be similar.

To examine the pattern of projections arising from different mediolateral positions within the same field of the entorhinal cortex, compare the terminal distributions of experiments DM23, DM-15, DM-32, and DM-13. Comparing the plots of terminal labeling in $c, f, g$, and $l$, it can be clearly seen that the pattern of innervation is heaviest in the uncal portion of the dentate gyrus in experiment DM-23, in the lateral part of the genu in experiment DM-15, in the rostral part of the body in DM-32, and in the caudal part of the body in case DM-13. Photomicrographs of the terminal labeling in the polar extremes of this comparison are illustrated in Figure 6. Terminal labeling in experiment DM-23 is heavy at rostral levels of the dentate gyrus $(A, B)$ but is absent at caudal levels $(C, D)$. In contrast,

Figure 5. Schematic representations of the distribution and density of anterograde labeling along the rostrocaudal axis of the dentate gyrus following 14 injections in the entorhinal and perirhinal cortices. Inset, Location and cxtent of the injections on an unfolded map of the entorhinal cortex (see text for details). 

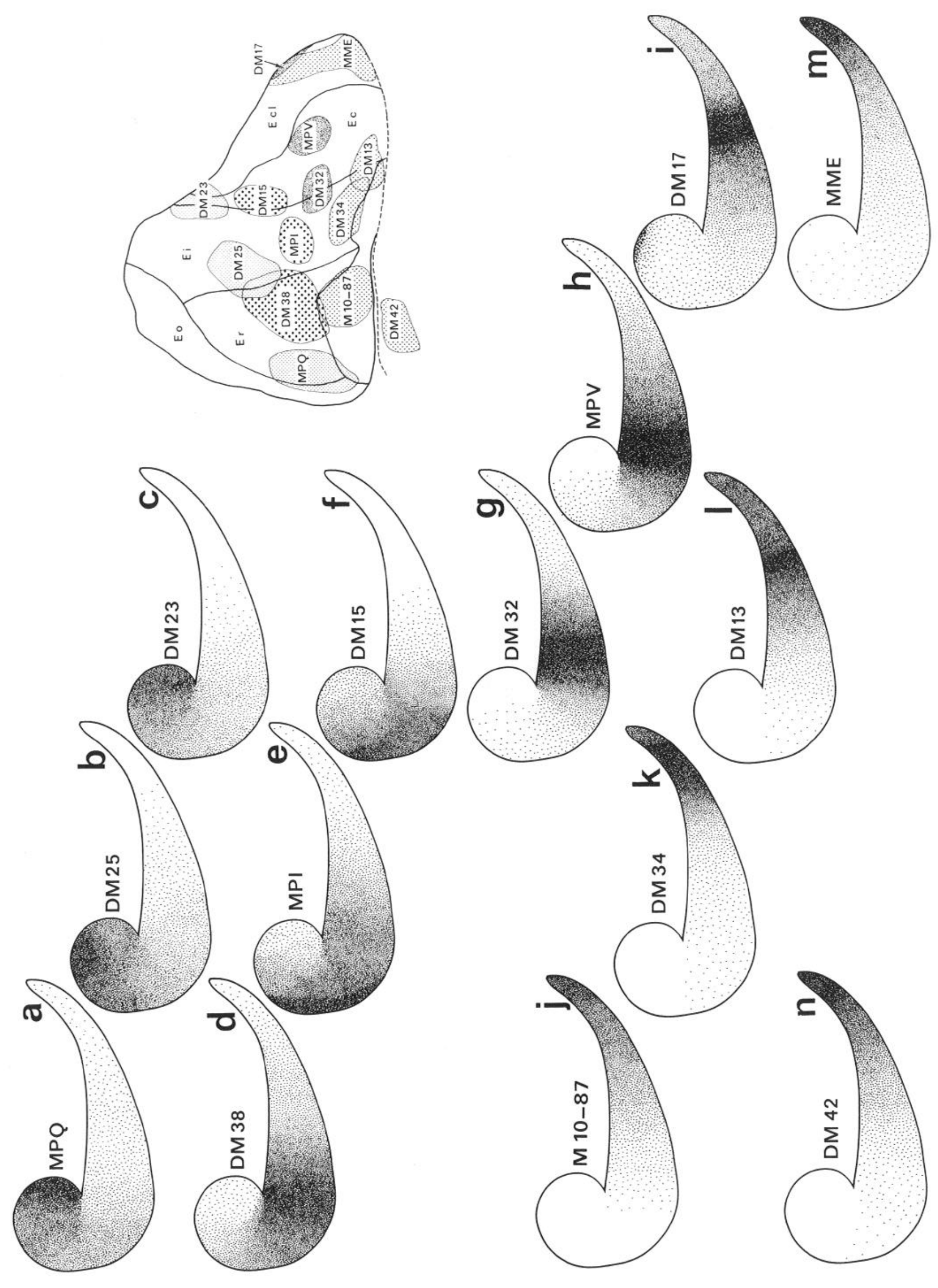

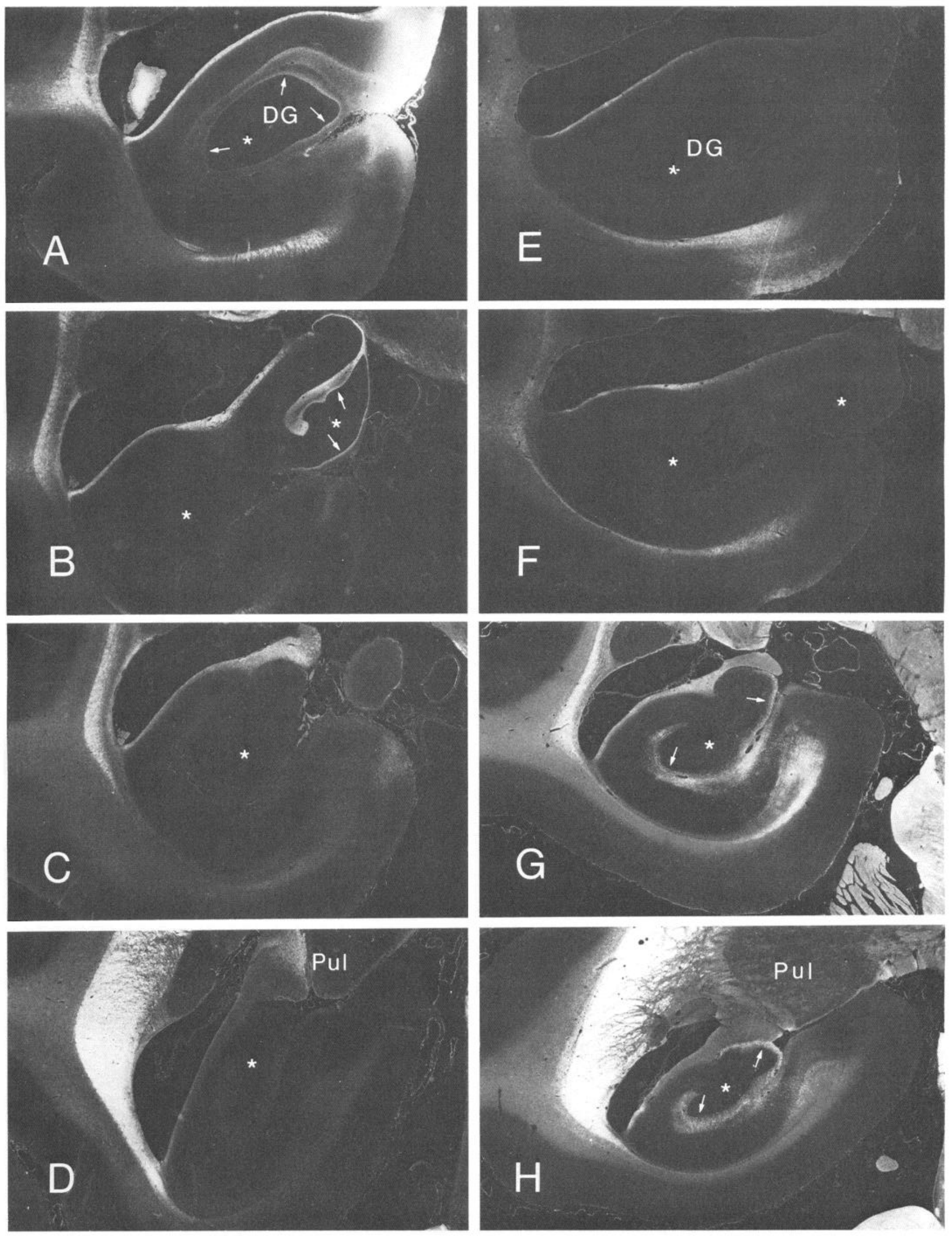
there is no labeling of the rostral dentate gyrus in experiment DM-13 $(E, F)$ but heavy labeling of the caudal levels $(G, H)$. A similar rostrocaudal shift of terminal labeling can be seen if experiments DM-25, MPI, and DM-34 are compared (Fig. 5, $b, e, k)$

The 14 experiments with ${ }^{3} \mathrm{H}$-amino acid injections can be combined into 4 groups based on their patterns of terminal labeling in the dentate gyrus. Taken together, the arcas cncompassed by the injection sites in each group form rostrocaudally oriented zones that are oblique to the rhinal sulcus and reminiscent of the zones of labeled cells found in the retrograde studies.

The first group includes experiments MPQ, DM-25, and DM23. While each of these injections is centered in a different subdivision of the entorhinal cortex, they nonetheless all label projections that terminate mainly in the uncal portion of the dentate gyrus. The second group includes experiments DM-38, MPI, and DM-15. Again, despite the fact that the injection sites range from Er to Ec, the pattern of terminal labeling, which is focused in the lateral part of the genu and the most rostral portion of the body of the dentate gyrus, is very similar in the 3 cases. The third group of cases includes experiments DM-32, MPV, and DM-17. The injections in these cases are situated caudally in the entorhinal cortex and approximately midway along the transverse axis. While labeling is fairly extensive in these cases, the region of highest density of termination in the intermediate portion of the body of the dentate gyrus is similar in all 3 experiments. Finally, there is a group of experiments in which the injections were located far laterally in the entorhinal cortex adjacent to the rhinal sulcus. This group includes experiments M-10-87, DM-34, DM-13, and MME. In each of these cases the density of terminal innervation is heaviest in the caudal part of the body of the dentate gyrus. Thus, the pattern of labeling in these cases is similar despite the fact that the positions of the injections range from nearly the rostral pole of the entorhinal cortex (M-10-87) to its caudal pole (MME).

As a final note, we should point out that labeled cells were observed in area 35 (perirhinal cortex) in the retrograde tracer experiments where the injection involved the caudal dentate gyrus. We had 3 autoradiographic cases available for analysis that had ${ }^{3} \mathrm{H}$-amino acid injections in area 35 or the adjacent area 36. A representative experiment (DM-42) is illustrated in Figure 5. This injection resulted in a pattern of anterograde labeling in the dentate gyrus that is comparable to those of the most lateral group of entorhinal injections. Our injections in area 36 did not produce labeling in the dentate gyrus, though there were prominent projections to the subiculum.

\section{Discussion}

Previous anterograde studies clearly demonstrated that in the monkey, as in the rat and cat, the entorhinal cortex gives rise to a prominent projection to the dentate gyrus and hippocampus (Van Hoesen and Pandya, 1975b; Van Hoesen, 1982). However, the laminar and topographic organization of the perforant pathway projection was not determined in these early studies.
Thus, in the present series of experiments we sought to determine which entorhinal cell layers give rise to the perforant pathway projections, as well as which regions of the entorhinal cortex project to different rostrocaudal levels of the dentate gyrus. These studies were designed in the context of a recent reevaluation of the cytoarchitectonic organization of the monkey entorhinal cortex (Amaral et al., 1987) and were based on newly formulated conccpts of the topographic organization of the perforant pathway projection that were derived from anterograde and retrograde studies in the cat (Witter and Groenewegen, 1984; Witter et al., 1986).

In relation to the laminar origin of the entorhinal projections to the dentate gyrus and hippocampus, there is substantial information available from studies conducted in the rat and cat (Steward and Scoville, 1976; Witter and Groenewegen, 1984; Köhler, 1985). The general conclusion to be drawn from these studies is that the projection to the dentate gyrus arises mainly from cells in layer II, but a minor component may also originate from deep cells. The projections to the subiculum and hippocampus, in contrast, originate in the deeper layers of the entorhinal cortex. Our results in the monkey are consonant with those in the nonprimates. Retrograde tracer injections that involved only the dentate gyrus resulted in labeling that was mainly confined to layer II, although at least some cells were generally seen in layer $\mathrm{V}$ as well. When the tracer injection involved either the hippocampus or the subiculum, in addition to the dentate gyrus, labeled cells were distributed more widely in the entorhinal cortex and occupied layers III, V, and VI, as well as layer II. Thus, the entorhinal projection to the dentate arises mainly from layer II, whereas deeper layers contribute projections that terminate in the hippocampus and subiculum.

We have not provided a detailed description of the laminar termination of the entorhinal projection to the dentate molecular layer, though this will form the major portion of a subsequent paper on the monkey perforant pathway. In nonprimates a lateral and a medial perforant pathway have been differentiated that terminate in the outer and middle thirds of the molecular layer, respectively (Steward, 1976; Wyss, 1981; Witter and Groenewegen, 1984). Not only do these pathways have differential terminal distributions, but in the rat they also appear to be physiologically distinct (McNaughton, 1980). The laminar organization that emerges from the current anterograde experiments in the monkey departs markedly from the pattern seen in nonprimates. While, as in the rat and cat, the monkey perforant pathway projection terminates mainly in the outer twothirds of the molecular layer, there is no clear-cut division of injections that lead to cither outer or middle third labeling. In each experiment, and regardless of the size of the injection, labeling in the molecular layer, at the level of the dentate gyrus that is most heavily innervated, tends to be distributed throughout the outer two-thirds. Only with injections that are confined to the most rostrolateral parts of the entorhinal cortex is a terminal field observed that is mainly restricted to the outer third of the molecular layer. Even in those cases, however, there is also weak labeling of the middle third. In all other cases, the

Figure 6. Dark-field photomicrographs of the dentate gyrus and hippocampus showing the distribution of anterograde labeling (arrowheads) at 4 different rostrocaudal levels in cases DM-23 $(A-D)$ and DM-13 $(E-H)$. Both injections are located on the border between Ei and Ec, but DM-23 was medially placed, while DM-13 was located laterally, near the rhinal sulcus. Closely matched sections are arranged from rostral $(A, E)$ to caudal $(D, H)$. Dense labeling is present at rostral levels in case DM-23 $(A, B)$, while no labeling is present at caudal levels $(C, D)$. The reverse is true in case DM-13 $(E-H)$. The dentate gyrus is indicated by asterisks. $\times 10.5$. 


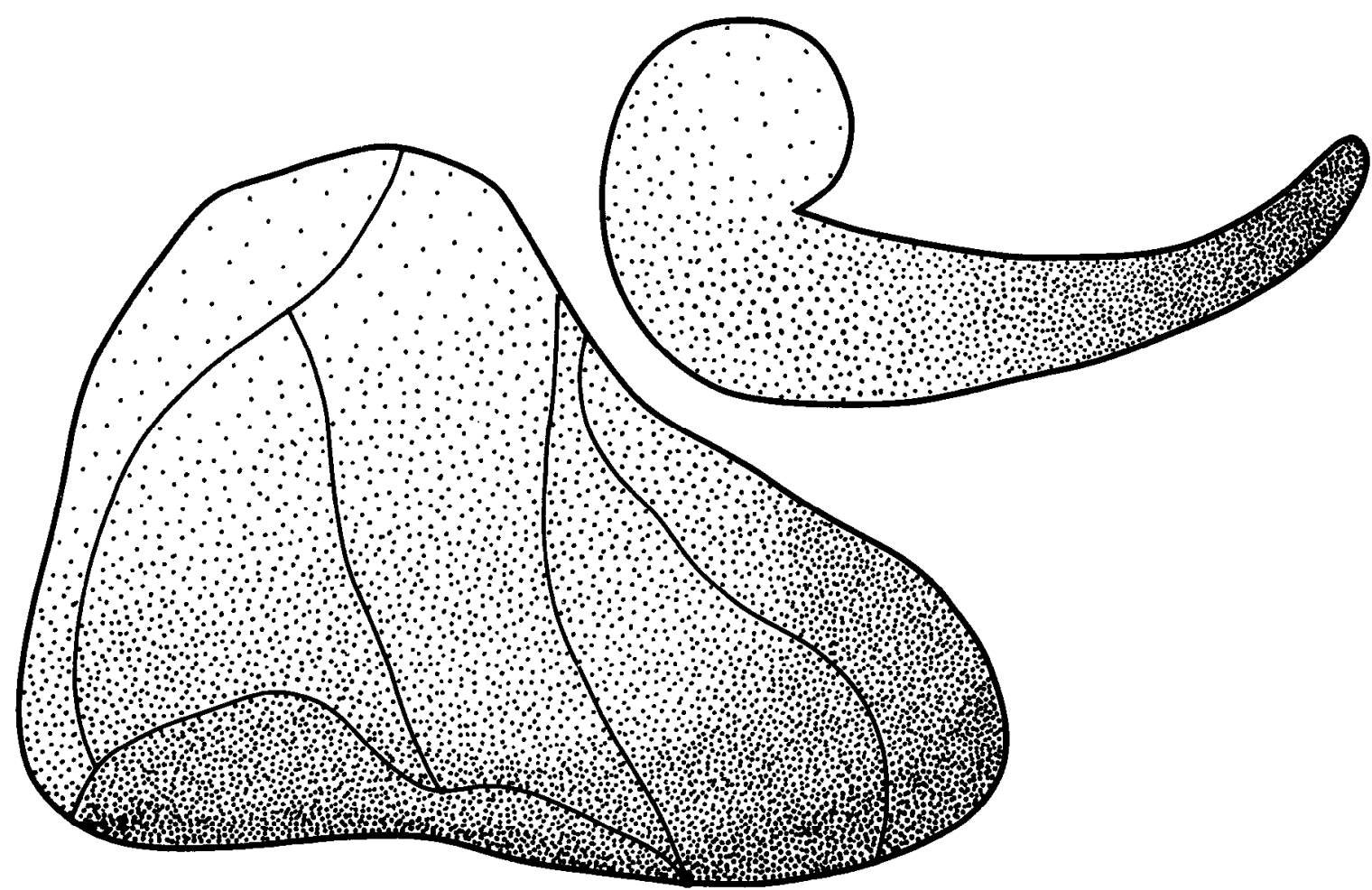

Figure 7. Topographic organization of the perforant pathway projection to the dentate gyrus is schematically illustrated. The origin and termination of the perforant pathway are indicated by corresponding density of stippling in the unfolded map of the entorhinal cortex (left) and the template of the dentate gyrus (right).

projection distributes throughout the entire outer two-thirds of the molecular layer, though the projections arising rostrally tend to terminate in the outer third and the more caudally arising fibers distribute preferentially to the middle third (see also Van Hoesen and Pandya, 1975b).

Perhaps the principal goal of the current series of studies was to determine the topographic organization of the monkey perforant pathway projection. The experiments described in this study were also conducted for the purpose of determining whether there is a point-to-point mapping of the entorhinal cortex onto the dentate gyrus or whether the perforant pathway projection is divergent. Electrophysiological data on the intrinsic circuitry of the hippocampal formation suggested that the connections from the dentate gyrus to the subiculum, via CA3 and $\mathrm{CA} 1$, are preferentially organized in narrow slices that are oriented essentially perpendicular to the long or septotemporal axis of the hippocampus (Andersen et al., 1971). The entorhinal projection was considered to respect this "lamellar" scheme in that small regions of the cortex were thought to map exclusively onto individual hippocampal lamellae. However, more recent anatomical evidence in the rat and cat (Wyss, 1981; Witter and Groenewegen, 1984) indicates that restricted parts of the entorhinal cortex project to a large septotemporal extent of the dentate gyrus. In the cat, where it has been most thoroughly studied (Witter and Groenewegen, 1984), it appears that rostrocaudally oriented zones of cells in the entorhinal cortex, which are arranged from lateral to medial, project to different septotemporal levels of the dentate gyrus; lateral cells project septally and more medially situated cells project progressively more temporally. Since the lateral portion of the cat entorhinal cortex is preferentially innervated by cortical afferents (Roum and Groenewegen, 1986; Witter et al., 1986), it appears that the septal portion of the dentate gyrus, compared with its temporal portion, has more immediate access to this route of cortical sensory information.

The major finding in the present series of experiments in the monkey is that projections from the entorhinal cortex to the dentate gyrus are organized in a manner nearly identical to that in the cat. The retrograde and anterograde experiments are consistent in indicating that the cells of origin of the cntorhinal projections to different rostrocaudal levels of the dentate gyrus are organized in obliquely oriented, longitudinal zones that more or less parallel the anterolateral-to-posteromedial axis in the entorhinal cortex (Fig. 7). We should point out that these longitudinal zones of cells do not appear to be sharply delimited. Even in the retrograde tracer experiments with 2 widely separated injections of fluorescent dyes (such as in experiment M-6-86 in which the 2 injections were separated by half the rostrocaudal distance of the dentate gyrus), the zones of retrogradely labeled cells were nonetheless partially overlapping and double-labeled cells occurred in the region of overlap. This indicates that most entorhinal neurons give rise to a rather divergent terminal distribution that involves a substantial portion of the rostrocaudal extent of the dentate gyrus. This conclusion is also substantiated by the anterograde tracing experiments. Whilc cach injection into the entorhinal cortex resulted in a fairly narrow zone of very dense labeling, even relatively small injections produced detectable labeling in at least one-third to one-half of the rostrocaudal extent of the dentate gyrus. Finally, since the major axis of cytoarchitectonic differentiation appears to be oriented rostrocaudally in the monkey entorhinal cortex (Amaral et al., 1987), the zones of cells that project to various rostrocaudal levels of the dentate gyrus are oriented orthogonal or cut across most of the entorhinal subdivisions. As a conse- 
quence, portions of several of the cytoarchitectonic divisions of the entorhinal cortex project together to different rostrocaudal levels of the dentate gyrus.

Given the clear-cut organization of the perforant pathway projection to the dentate gyrus, an obvious question is how this organization might influence the function of the hippocampal formation. It is fairly clear, for example, that if the lateral part of the entorhinal cortex received a particular type of input, that that input would more immediately influence the caudal portions of the dentate gyrus. Conversely, inputs to the rostromedial parts of the entorhinal cortex would have more impact on rostral levels of the dentate gyrus.

Although details concerning the termination of afferents to the entorhinal cortex in monkeys are meager, there are indications from both anterograde (Van Hoesen and Pandya, 1975a; Van Hoesen et al., 1975; Amaral et al., 1983) and retrograde (Insausti et al., 1987) studies that projections arising in several cortical regions, such as the perirhinal and parahippocampal cortices, the orbitofrontal cortex and portions of the superior temporal gyrus, preferentially terminate in the lateral and caudal portions of the entorhinal cortex. The preferential termination of cortical inputs in the lateral portion of the entorhinal cortex in the monkey is similar to the situation observed in the cat (Room and Groenewegen, 1986). It would appear, therefore, that caudal levels of the dentate gyrus would be more immediately influenced by cortical sensory information. It should be borne in mind, however, that the extensive associational projections of the dentate gyrus and hippocampus have the potential of widely dispersing the inputs that come into any particular level. There is far less information concerning the inputs to rostromedial portions of the entorhinal cortex. One obviously distinct projection is from the olfactory system. The olfactory portion of the entorhinal cortex receives a direct projection from the olfactory bulb, and this would be relayed to the most rostral levels of the dentate gyrus. Thus, the rostral dentate gyrus may be immediately or powerfully influenced by olfactory information. These findings, as well as various other lines of evidence (for a review of the relevant literature, see Witter, 1986; Witter et al., 1986), indicate that different rostrocaudal regions of the hippocampal formation may be functionally heterogeneous. It is certainly clear that an understanding of hippocampal function will bc aided by a precise terminal mapping of various inputs to the entorhinal cortex and an appreciation of how the entorhinal cortex relays the incoming information to other fields of the hippocampal formation.

\section{References}

Amaral, D. G., and W. M. Cowan (1980) Subcortical afferents to the hippocampal formation in the monkey. J. Comp. Neurol. 189: 573591 .

Amaral, D. G., and J. L. Price (1983) An air pressure system for the injection of tracer substances into the brain. J. Neurosci. Methods 9: $35-43$.

Amaral, D. G., R. Insausti, and W. M. Cowan (1983) Evidence for a direct projection from the superior temporal gyrus to the entorhinal cortex in the monkey. Brain Res. 275: 263-277.

Amaral, D. G., R. Insausti, and W. M. Cowan (1984) The commissural connections of the monkey hippocampal formation. J. Comp. Neurol. 224: 307-336.

Amaral, D. G., R. Insausti, and W. M. Cowan (1987) The entorhinal cortex of the monkey: I. Cytoarchitectonic organization. J. Comp. Neurol. 264: 326-355.

Andersen, P., T. V. P. Bliss, and K. K. Skrede (1971) Lamellar or- ganization of hippocampal excitatory pathways. Exp. Brain Res. 13: 222-238.

Cowan, W. M., D. I. Gottlieb, A. E. Henderson, J. L. Price, and T. A. Woolsey (1972) The autoradiographic demonstration of axonal connections in the central nervous system. Brain Res. 37: 21-55.

Deacon, T. W., H. Eichenbaum, P. Rosenberg, and K. W. Eckmann (1983) Afferent connections of the perirhinal cortex in the rat. J. Comp. Neurol. 220: 168-190.

Demeter, S., D. L. Rosene, and G. W. Van Hoesen (1985) Interhemispheric pathways of the hippocampal formation, presubiculum, and entorhinal and caudal parahippocampal cortices in the Rhesus monkey: The structure and organization of the hippocampal commissures. J. Comp. Neurol. 233: 30-47.

Insausti, R., D. G. Amaral, and W. M. Cowan (1987) The entorhinal cortex of the monkey: II. Cortical afferents. J. Comp. Neurol. 264: 356-395.

Jones, E. G., and T. P. S. Powell (1970) An anatomical study of converging sensory pathways within the cerebral cortex of the monkey. Brain 93: 793-820.

Köhler, C. (1985) A projection from the deep layers of the entorhinal area to the hippocampal formation in the rat brain. Neurosci. Lett. 56:13-19.

MeNaughton, B. L. (1980) Evidence for two physiologically distinct perforant pathways to the fascia dentata. Brain Res. 199: 1-19.

Mesulam, M. M. (1976) The blue reaction product in horseradish peroxidase histochemistry. Incubation parameters and visibility. $\mathbf{J}$. Histochem. Cytochem. 24: 1273-1280.

Mishkin, M. (1978) Memory in monkeys severely impaired by combined but not by separate removal of amygdala and hippocampus. Nature 273: 297-298.

Room, P., and H. J. Groenewegen (1986) Connections of the parahippocampal cortex. I. Cortical afferents. J. Comp. Neurol. 251:415450.

Ruth, R. E., T. J. Collier, and R. Routtenberg (1982) Topography between the entorhinal cortex and the dentate septotemporal axis in rats: I. Medial and intermediate entorhinal projecting cells. J. Comp. Neurol. 209:69-78.

Scoville, W. B., and B. Milner (1957) Loss of recent memory after bilateral hippocampal lesions. J. Neurol. Neurosurg. Psychiatry 20: $11-21$

Steward, O. (1976) Topographic organization of the projections from the entorhinal areas to the hippocampal formation of the rat. J. Comp. Neurol. 167: 285-314.

Steward, O., and S. A. Scoville (1976) Cells of origin of entorhinal cortical afferents to the hippocampus and fascia dentata of the rat. J. Comp. Neurol. 169: 347-370.

Szabo, J., and W. M. Cowan (1984) A stereotaxic atlas of the brain of the Cynomolgus monkey (Macaca fascicularis). J. Comp. Neurol. 222: $265-300$.

Van Groen, T., and F. H. Lopes da Silva (1985) Septotemporal distribution of entorhinal projections to the hippocampus in the cat: Electrophysiological evidence. J. Comp. Neurol. 238: 1-10.

Van Hoesen, G. W. (1982) The parahippocampal gyrus. New observations regarding its cortical connections in the monkey. TINS 5: 345-350.

Van Hoesen, G. W., and D. N. Pandya (1975a) Some connections of the entorhinal (area 28) and perirhinal (area 35) cortices of the Rhesus monkey. I. Temporal lobe afferents. Brain Res. 95: 1-24.

Van Hoesen, G. W., and D. N. Pandya (1975b) Some connections of the entorhinal (area 28) and perirhinal (area 35) cortices of the Rhesus monkey. III. Efferent connections. Brain Res. 95: 39-59.

Van Hoesen, G. W., D. N. Pandya, and N. Butters (1975) Some connections of the entorhinal (area 28) and perirhinal (area 35) cortices of the Rhesus monkey. II. Frontal lobe afferents. Brain Res. 95: $25-38$

Witter, M. P. (1986) A survey of the anatomy of the hippocampal formation, with emphasis on the septotemporal organization of its intrinsic and extrinsic connections. Adv. Exp. Med. Biol. 23: 67-82.

Witter, M. P., and H. J. Groenewegen (1984) Laminar origin and septotemporal distribution of entorhinal and perirhinal projections to the hippocampus in the cat. J. Comp. Neurol. 224: 371-385.

Witter, M. P., P. Room, H. J. Groenewegen, and A. H. M. Lohman (1986) The connections of the parahippocampal cortex in the cat. $\mathrm{V}$. Intrinsic connections; Comments on input/output connections with the hippocampus. J. Comp. Neurol. 252: 78-94. 
Wyss, J. M. (1981) An autoradiographic study of the efferent connections of the entorhinal cortex in the rat. J. Comp. Neurol. 199:495512.

Zola-Morgan, S., and L. R. Squire (1986) Memory impairment in monkeys following lesions limited to the hippocampus. Behav. Neurosci. 100: 155-160.
Zola-Morgan, S., L. R. Squire, and D. G. Amaral (1986) Human amnesia and the medial temporal region: Enduring memory impairment following a bilateral lesion limited to field CAI of the hippocampus. J. Neurosci. 6: 2950-2967. 